\title{
Itinéraires
}

Itinéraires Littérature, textes, cultures

2010-3 | 2010

Médiévalisme

\section{Jane Chance (ed.), Tolkien the Medievalist}

New York et Londres, Routledge, coll. « Routledge Research in Medieval Religion and Culture », 2003, xv, 295 p.

\section{Marguerite Mouton}

\section{(2) OpenEdition}

1 Journals

Édition électronique

URL : http://journals.openedition.org/itineraires/1914

DOI : 10.4000/itineraires.1914

ISSN : 2427-920X

\section{Éditeur}

Pléiade

\section{Édition imprimée}

Date de publication : 1 novembre 2010

Pagination : 192-196

ISBN : 978-2-296-13150-7

ISSN : 2100-1340

\section{Référence électronique}

Marguerite Mouton, « Jane Chance (ed.), Tolkien the Medievalist », Itinéraires [En ligne], 2010-3 | 2010, mis en ligne le 01 novembre 2010, consulté le 22 septembre 2020. URL : http://

journals.openedition.org/itineraires/1914; DOI : https://doi.org/10.4000/itineraires.1914

Ce document a été généré automatiquement le 22 septembre 2020.

\section{(c)}

Itinéraires est mis à disposition selon les termes de la licence Creative Commons Attribution - Pas d'Utilisation Commerciale - Pas de Modification 4.0 International. 


\section{Jane Chance (ed.), Tolkien the Medievalist}

New York et Londres, Routledge, coll. « Routledge Research in Medieval Religion and Culture », 2003, xv, 295 p.

\section{Marguerite Mouton}

\section{RÉFÉRENCE}

Jane Chance (ed.), Tolkien the Medievalist, New York et Londres, Routledge, coll.

« Routledge Research in Medieval Religion and Culture », 2003, xv, 295 p.

1 Jane Chance, professeure à l'université de Rice (Texas), a publié à ce jour une vingtaine de livres. Tolkien the Medievalist se situe au croisement de ses deux domaines de recherche. J. Chance a en effet publié d'une part des ouvrages critiques centrés sur les mythes médiévaux, tels les trois volumes rassemblés sous le titre de Medieval Mythography (1994, 2000, 2006), ou encore sur la réception moderne du Moyen Âge, explorée à travers le tome de la collection Studies in Medievalism $(1982,1991)$ dont elle a supervisé l'édition. D'autre part, elle s'est intéressée au groupe des Inklings, et en particulier à l'œuvre de J. R. R. Tolkien, à laquelle elle a consacré Tolkien's Art: A Mythology for England $(1979,2001)$ et The Lord of the Rings: The Mythology of Power (1992, 2001). Un troisième champ de recherche se dessine en 2003 dans l'édition de Tolkien the Medievalist, celui de l'étude des figures féminines. Celui-ci fera l'objet de deux ouvrages ultérieurs de J. Chance, mais apparaît déjà dans l'article de Leslie A. Donovan intitulé "The valkyrie reflex in J. R. R. Tolkien's The Lord of the Rings: Galadriel, Shelob, Eowyn and Arwen ».

2 L'édition de Tolkien the Medievalist contient une introduction (p. 1-12), quinze articles dont dix ont été présentés au 36e Congrès international d'études médiévales de Kalamazoo en 2001, une longue bibliographie (p. 268-284) et un index (p. 285-295). D'un point de vue thématique, le recueil semble s'inscrire dans la lignée des ouvrages antérieurs de Jane Chance ; pourtant, l'introduction du volume présente celui-ci comme 
un événement dans l'histoire de la critique tolkienienne. Il s'agit de développer des «moyens nouveaux et mieux informés » pour lire l'œuvre (p.4). Il est toutefois difficile d'admettre sans discussion que le rapport de Tolkien au Moyen Âge souffrirait d'un grave manque d'analyses sérieuses, d'autant que J. Chance rappelle un certain nombre de grandes études, dont celle qu'elle-même a consacrée à la question de la création tolkienienne d'une "mythologie pour l'Angleterre », ou encore le magistral ouvrage de Tom Shippey, The Road to Middle-earth $(1982,1992)$. Les contributeurs de l'ouvrage de J. Chance éprouvent d'ailleurs une certaine difficulté à proposer de nouveaux outils, malgré le choix d'étendre la période historique de référence depuis Saint Augustin jusqu'à la fin du xvie siècle. Ils ont tendance à s'appuyer sur les notions forgées par Tom Shippey, telles la "réalité astérisque» ou le concept de "profondeur» des histoires, qu'ils se contentent souvent d'appliquer à d'autres exemples.

3 Néanmoins, J. Chance rappelle que la parution de manuscrits inédits de Tolkien depuis les ouvrages critiques du début des années 1980 (les douze volumes de History of Middleearth entre 1983 et 1996 et Roverandom en 1998) offre des horizons inexplorés à la critique. Cette considération suffirait à justifier l'entreprise si les articles qui composent l'ouvrage accordaient effectivement une place essentielle à l'analyse des dernières publications. Mais de telles études semblent circonscrites à la quatrième partie. Celle-ci explore en effet l'apport des différentes versions des textes composant le Légendaire à l'interprétation d'histoires telles que celles de Túrin ou encore de Beren et Lúthien.

4 Ce qui rend l'ouvrage dirigé par J. Chance particulièrement opportun en 2003, c'est avant tout le succès mondial du film de Peter Jackson, et l'explosion des ventes des livres de Tolkien à cette occasion. L'introduction de Jane Chance juxtapose ainsi des considérations sur la réception de l'œuvre de Tolkien et d'autres sur l'importance du Moyen Âge dans la conception du texte, et va presque jusqu'à revendiquer un lien de causalité entre les deux. L'affirmation de Chance selon laquelle il convient de fournir au lecteur des «moyens nouveaux et mieux informés » retrouve toute sa pertinence dans le contexte de cette massification de la réception de l'œuvre et d'une éventuelle baisse de la qualité de la critique.

5 C'est bien à ce besoin que semblent répondre les articles de la première partie de l'ouvrage, qui offrent un éclairage biographique ou traitent de la question (parfois présentée sous un jour polémique) du rapport de l'auteur à l'idéologie nazie. Dans son article (" "An industrious little devil": E. V. Gordon as friend and collaborator with Tolkien »), Douglas A. Anderson, éminent connaisseur et éditeur des textes de Tolkien, se penche sur la relation de Tolkien et de son collaborateur et successeur à la chaire de Leeds, E. V. Gordon. Il rapporte d'intéressants éléments concernant le tempérament de Tolkien mais aussi ses travaux scientifiques sur les textes médiévaux que sont Sir Gawain and the Green Knight et Pearl. En revanche, dans un article intitulé «A kind of mid-wife : J. R. R. Tolkien and C. S. Lewis - sharing influence ", Andrew Lazo, alors doctorant à l'université de Rice, propose davantage une synthèse de faits connus sur les influences communes et mutuelles de Tolkien et de C. S. Lewis. Il revient ainsi sur la discussion nocturne de 1931 qui marque la conversion de Lewis à la foi chrétienne, mais aussi sur les goûts des deux hommes pour la nature, la littérature nordique, Homère et le romance médiéval.

Le commentaire de Verlyn Flieger sur la célèbre conférence de Tolkien intitulée « Du Conte de fées » et le long article de Christine Chism consacré au rapport de l'auteur au 
nazisme concentrent l'attention sur le débat idéologique contemporain de la rédaction. Dans son étude (" There would always be a fairy-tale : J. R. R. Tolkien and the folklore controversy »), V. Flieger défend l'idée que si Tolkien minimise l'importance de l'origine dans sa réflexion sur le conte de fées, c'est sans doute parce que le contexte historique tend à associer cette question à la constitution d'une identité aryenne. Pour sa part, Christine Chism examine la manière dont Tolkien, loin de nier tout rôle de la question de l'aryanisme dans la conception de son œuvre, s'efforce de justifier la production d'un monde imaginaire à partir d'une mythologie nordique qui inspire à la même époque l'entreprise nazie. L'article défend la thèse selon laquelle Le Seigneur des Anneaux peut être interprété comme une réponse de Tolkien à cette question, dans la mesure où l'œuvre ferait le récit de la sortie du mythe et de l'avènement de l'histoire grâce au renoncement symbolique à l'Anneau de Sauron. En proposant une analyse interne du fonctionnement de l'œuvre, C. Chism évite de s'exposer frontalement au reproche traditionnel de faire du Seigneur des Anneaux une allégorie de la situation politique contemporaine, à condition toutefois de ne pas céder à la tentation qui la guette d'affirmer que le texte de Tolkien constitue une réponse directe au débat idéologique de son temps.

7 Mary Faraci, professeure à l'université de Floride, revient sur une autre conférence de Tolkien. Reprenant l'opposition que celui-ci établit entre les critiques qui imposent leur mode de lecture au poème Beowulf et ceux qui étudient l'œuvre de l'intérieur, selon les critères du poète lui-même, Mary Faraci propose d'interpréter cette différence sur le modèle de la distinction qui existe entre voie active et voie moyenne dans la grammaire du grec ancien. L'analyse des deux types de critique décrits par Tolkien ne manque pas d'intérêt, mais le recours aux outils de la linguistique antique s'appuie sur peu de données textuelles, et l'article court le risque de reproduire l'attitude des critiques qui imposent leur propre grille de lecture à un texte qui, certes, n'est pas un poème, mais partage certains traits du texte du poète de Beowulf avec lequel il entend développer une relation empathique.

8 Les deuxième et troisième sections de Tolkien the Medievalist quittent l'éclairage de la réception contemporaine du Moyen Âge pour une série de comparaisons ponctuelles très spécialisées entre des textes ou motifs médiévaux et des épisodes ou personnages de l'œuvre de Tolkien. Sont convoquées les imageries traditionnelles de l'Homme Sauvage, de l'Armée des morts ou encore de la Vierge Marie telle qu'elle apparaît dans les litanies du Xvi ${ }^{e}$ siècle. Certaines comparaisons sont plus fructueuses que d'autres. L'apport de la tradition de la "musique des sphères » à l'analyse du Silmarillion laisse perplexe. Bradford Lee Eden peine d'ailleurs à trouver un équivalent textuel à la musique instrumentale qui est censée cohabiter avec la musique de l'univers et la musique vocale ("The "music of the spheres" : relationships between Tolkien's The Silmarillion and medieval cosmological and religious theory »). D'autres comparaisons n'apportent pas beaucoup à la réflexion sur l'œuvre. L'article de Jonathan Evans, «The anthropology of Arda: creation, theology and the race of Men », ne fait qu'étoffer l'analyse de Tom Shippey, reprenant par exemple sa comparaison entre Le Silmarillion et Le Paradis perdu de Milton. En revanche, John William Houghton («Augustine in the cottage of lost play : the Ainulindalë as asterisk cosmogony ») établit à nouveaux frais la compatibilité de l'« Ainulindalë » avec les autres traditions des récits de création du haut Moyen Âge, en examinant avec soin les points de jonction et les écarts entre le texte de Tolkien et les commentaires augustiniens de la Genèse. 
L'article de L. A. Donovan propose enfin une analyse très intéressante des personnages féminins du Seigneur des Anneaux à l'aune de la figure traditionnelle de la valkyrie. Il est seulement dommage qu'après avoir souligné l'importance de ne pas lire l'œuvre de Tolkien selon les critères modernes mais à l'aide des outils d'analyse des textes médiévaux, L. A. Donovan ne puisse s'empêcher de conclure que Tolkien fait preuve d'une grande "largeur de vues" et d'une ouverture à de "nouveaux modèles familiaux » propres à le racheter aux yeux du féminisme (p. 109).

Il reste à signaler dans la quatrième partie du volume, consacrée à l'influence de phénomènes d'écriture typiquement médiévaux sur l'œuvre de Tolkien, le remarquable article de Gergely Nagy, alors doctorant en Hongrie, qui analyse "The great chain of reading: (inter-)textual relations and the technique of mythopoesis in the Túrin story ». L'auteur reprend la notion de "profondeur» des histoires forgée par Tom Shippey et développe tout un dispositif conceptuel d'analyse du fonctionnement du texte tolkienien, qu'il applique à l'exemple de Túrin. Le Légendaire fonctionnerait dans le monde secondaire sur le modèle de la mythologie dans le monde primaire. L'œuvre de Tolkien crée un tissu de textes et d'histoires plus ou moins accessibles au lecteur mais qui renvoient les uns aux autres, de la même manière que les textes du poète de Beowulf, de Malory ou de Chaucer convoquent des références et les mettent en réseau. La création tolkienienne reproduirait donc pour le lecteur les conditions de la réception actuelle des textes médiévaux, dont il ne peut saisir toutes les références mais dont il ressent la « profondeur » mythologique.

Le recueil se clôt sur une analyse brève mais très informée de l'histoire de Beren et Lúthien. Dans un article intitulé « Real-world myth in a secondary world : mythological aspects in the story of Beren and Lúthien", Richard C. West étudie les différentes versions de l'histoire et les influences biographiques, littéraires et mythologiques qui la traversent. On peut seulement regretter le manque de références précises, comme si, en dernier ressort, le lecteur de Tolkien the Medievalist était finalement invité à retrouver une lecture plus empathique qu'érudite de l'œuvre du Professeur. Ces deux derniers articles achèvent avec brio un volume qui, par ailleurs, relève de manière inégale le délicat défi qu'il s'était lancé de développer des « moyens nouveaux et mieux informés » pour lire l'œuvre de Tolkien à la lumière du Moyen Âge.

\section{BIBLIOGRAPHIE}

Chance (Nitzsche), Jane, Tolkien's Art : A Mythology for England, London, Macmillan, 1979 ; Lexington, University Press of Kentucky, 2001 (éd. révisée).

Chance, Jane, The Lord of the Rings: The Mythology of Power, New York, Twayne/Macmillan, 1992; Lexington, University Press of Kentucky, 2001 (éd. révisée).

-, Medieval Mythography, Gainesville, University Press of Florida - Medieval Mythography: From Roman North Africa to the School of Chartres, 1994 (vol. 1) ; Medieval Mythography : From the School of 
Chartres to the Court at Avignon, AD 1177 to 1350, 2000 (vol. 2) ; Medieval Mythography: The Italian Renaissance, AD 1350-1478, 2006 (vol. 3).

- (ed.), Medievalism in the Twentieth Century, Studies in Medievalism, 1982, réédition par Boydell and Brewer sous le titre The Inklings and Others, Studies in Medievalism, 1991.

\section{AUTEURS}

\section{MARGUERITE MOUTON}

Université Paris 13-Paris Nord (CENEL) 